\title{
Factores determinantes del empleo informal en la región Moquegua
}

Determining factors of informal employment in the Moquegua region

Wilson Cañapatana Castillo y Deysi Diana Quipe Flores

Universidad Nacional de San Agustín, Arequipa Perú.

INFORMACIÓN

\section{Historia del Artículo}

Recepción: 15/05/2019

Revisión: 02/07/2019

Aceptación: 12/10/2019

\section{Palabras Clave}

Factores determinantes, Empleo informal, Región Moquegua, Modelo $\operatorname{logit}$

\section{Key Words \\ Determining factors, Informal employment, Moquegua región, Logit model.}

\section{DOI}

https://doi.org/10.35286/veritas. v21i1.256

\begin{abstract}
RESUMEN
El empleo informal se ha convertido en un problema social, pues gran parte de la población no tiene acceso a las condiciones más favorables de trabajo. El empleo informal conlleva a perjuicios para el desarrollo económico y bienestar de la sociedad, pues posibilita la falta de acceso a los beneficios laborales, como acceso a un seguro de salud, gratificaciones y vacaciones. En este trabajo se presenta un modelo logit de probabilidad no lineal, el cual es uno de los modelos más usados para determinar los factores más importantes en variables de respuesta binaria. En tal sentido la finalidad de este trabajo es contribuir con la comunidad local y científica mediante la identificación de los factores que determinan el empleo informal en la región. Los resultados del presente trabajo indican que el ser mujer, pertenecer al sector servicios, comercio, construcción o industria, contar con educación secundaria o superior, ser joven y provenir del área rural son características que determinan la probabilidad de obtener un empleo informal en la región Moquegua.
\end{abstract}

\begin{abstract}
Informal employment has become a social problem, since a large part of the population does not have access to the most favorable working conditions. Informal employment leads to damages for the economic development and welfare of society, since it makes possible the lack of access to labor benefits, such as access to health insurance, perks and vacations. In this paper, a logit model of nonlinear probability is presented, which is one of the most used models to determine the most important factors in binary response variables. In this sense, the purpose of this work is to contribute with the local and scientific community by identifying the factors that determine informal employment in the region. The results of this work indicate that being a woman, belonging to the services, commerce, construction or industry sector, having secondary or higher education, being young and coming from the rural area are characteristics that determine the probability of obtaining informal employment in the region Moquegua
\end{abstract}

\section{INTRODUCCIÓN}

Desde hace ya varios años que la informalidad viene llamando la atención como una de las posibles barreras al crecimiento económico y al bienestar social, así mismo se concibe como una fuerza que corroe la integridad de las sociedades en Latinoamérica (Banco Mundial, 2007).

Desde otra perspectiva el empleo informal hace referencia a aquellos empleos que no gozan de beneficios estipulados por ley como seguridad social, gratificaciones, vacaciones pagadas, etc. Hasta el año 2012 en el Perú aproximadamente el $74 \%$ de trabajadores que formaban parte de la PEA ocupada se encontraban en un empleo informal. El $57 \%$ lo hacía en un empleo informal dentro del sector informal y el restante $17 \%$ en un empleo informal fuera del sector informal (INEI, 2014). Así, mismo para el año 2016, el $72 \%$ de los trabajadores tuvo un empleo informal. Esto es cerca de 11 millones 657 mil 322 personas que laboran en el sector informal o empleos asalariados fuera del sector informal sin protección social, esto incluía a los trabajadores familiares no remunerados. El 55,0\% de la PEA ocupada del

Correspondencia:

Wilson Cañapatana castillo

wilsoncancas@gmail.com
Perú trabajó de manera informal en el sector informal y el $17,0 \%$ fuera del sector informal. Esto significa que el empleo informal se concentró en aquellas unidades productivas o empresas que no se encontraron registradas en SUNAT. En los últimos diez años esta cifra alcanzó un promedio anual de 75\% (MTPE, 2016).

Según la ENAHO-2014, uno de los sectores que dinamiza la economía nacional es el sector construcción, el cual fue uno de los sectores más informales en la zona norte del país. En el caso de la región Moquegua la informalidad laboral para el año 2014 alcanzo el 67\%, siendo una de las más bajas a nivel nacional (CEPLAN, 2016).

Al considerarse la informalidad laboral una barrera al crecimiento económico y bienestar social, entonces es de gran importancia determinar cuáles son los factores que influyen en la informalidad laboral en la región Moquegua.

"El empleo informal comprende el número total de empleos informales (...), ya se ocupen éstos en empresas del sector formal, empresas del sector informal, o en hogares, durante un período de referencia determinado" (OIT 2003, inciso 3.1).

Por otra parte, el Banco mundial señala que el empleo informal abarca una amplia gama de personas. En tal sentido ante la existencia de diferentes criterios tradicionales de definir el empleo informal, se presta una especial atención a la definición relacionada con el acceso a la protección social. Es decir, tiene en cuenta a los trabajadores asalariados que no 
se encuentran registrados en el sistema de seguridad social. Esta definición comprende a dos grupos:

i) Los trabajadores informales independientes

ii) Los trabajadores informales asalariados

"El sector independiente informal incluye a los propietarios de microempresas y a los profesionales independientes, así como a los artesanos, trabajadores del bricolaje, obreros de la construcción, choferes de taxis, y vendedores callejeros. El sector informal asalariado abarca en gran medida a los empleados domésticos, trabajadores familiares sin sueldo, trabajadores de microempresas, y los que trabajan en empresas más grandes bajo arreglos laborales informales" (Banco Mundial, 2007).

Así mismo el INEI señala que el empleo informal está referido al total de empleos que cumplen con ciertas condiciones, según la categoría de ocupación del trabajador:

i) Los patronos y cuenta propia cuya unidad productiva pertenece al sector informal.

ii) Los asalariados sin seguridad social financiada por su empleador.

iii) Los trabajadores familiares no remunerados, independientemente de la naturaleza formal o informal de la unidad productiva donde labora.

Esta investigación se centra en la región Moquegua tomando en cuenta como periodo de estudio el año 2016. El propósito de esta investigación es determinar aquellas variables socioeconómicas que son determinantes en el empleo informal en la región Moquegua, de manera que ayude a formular políticas sociales direccionadas a mejorar la situación informal de la población.

En tal sentido el objetivo es identificar los factores determinantes del empleo informal en la región Moquegua mediante un análisis cuantitativo de variables socioeconómicas.

\section{MATERIALES Y MÉTODOS}

En esta investigación se emplea el enfoque cuantitativo. Así mismo el diseño de esta investigación es descriptiva, correlacional y explicativa. Esto debido a que en la investigación se describen algunas variables, se asocian variables y se explica su comportamiento en el modelo. En lo que se refiere a la dimensión de la investigación, esta es de corte transversal ya que se tomaron muestras de las variables para diferentes individuos de la región Moquegua en el año 2016.

La población objetivo de este estudio está constituido por los trabajadores que forman parte de la Población Económicamente Activa y que cuentan con un empleo informal dentro de la región Moquegua para el año 2016. Por tal motivo se ha hecho uso de los datos recolectados por el INEI en la Encuesta Nacional de Hogares 2016, que a su vez es tratada por el Ministerio de Trabajo y Promoción del Empleo. Es decir que se trabaja con el módulo de empleo e ingresos de la Encuesta Nacional de Hogares trabajada por el Ministerio de Trabajo y Promoción del Empleo y los módulos de características de los miembros del hogar, educación y salud de la Encuesta nacional de Hogares del INEI.

Así mismo, dentro de la literatura económica existen una variedad de métodos a aplicar para determinar el efecto de una variable en otra, en este caso se trata de la probabilidad de un individuo de formar parte del empleo informal. Dentro de esta clase de modelos de variable dependiente binaria podemos encontrar los modelos de probabilidad lineal, modelos de regresión Tobit, modelos de regresión Logít y modelos de regresión Probit, siendo estos últimos tres modelos de probabilidad no lineal.

En algunas ocasiones se tienen dificultades en el modelo de probabilidad lineal, ya que la probabilidad estimada por este modelo no se encontraría acotado entre 0 y 1 , lo que llevaría a tener probabilidades negativas y probabilidades mayores al $100 \%$. En tal sentido es recomendable hacer el uso de un modelo Logit o Probit, los cuales proveen de probabilidades estimadas que se encuentran acotadas entre 0 y 1 . En el caso de los modelos Logit y Probit sin embargo las diferencias se dan principalmente en la función de distribución acumulada que emplea cada una de ellas (Stock \& Watson, 2012).

En este sentido, debido a las características de la población, se empleará un modelo Logit para explicar la probabilidad de formar parte del empleo informal. Dicho modelo se explica a continuación.

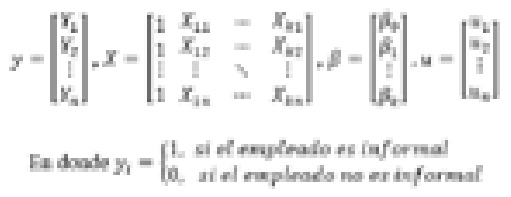

X1i : nativo, donde tomará el valor de 1 si la lengua materna del individuo es quechua, aymara o alguna otra lengua nativa del Perú y 0 si habla castellano o algún idioma extranjero.

$\mathrm{X} 2 \mathrm{i}$ : mujer, donde tomará el valor de 1 si el individuo es mujer y 0 si es hombre.

X3i : hogar, donde esta variable tomará el valor de 1 si el individuo es jefe de hogar y 0 si no lo es ${ }^{3}$.

$\mathrm{X} 4 \mathrm{i}$ : servicio, donde tomará el valor de 1 si el individuo se ubica laborando en el sector económico de servicio y 0 si se ubica en otro sector o rama de actividad económica.

X5i : comercio, donde esta variable tomará el valor de 1 si el individuo se ubica laborando en el sector económico de comercio y 0 si se ubica en otro sector o rama de actividad económica.

X6i : construcción, donde tomará el valor de 1 si el individuo se ubica laborando en el sector económico de construcción y 0 si se ubica en otro sector o rama de actividad económica. 
X7i : industria, donde esta variable tomará el valor de 1 si el individuo se ubica laborando en el sector económico de industria y 0 si se ubica en otro sector o rama de actividad económica.

X8i : superior, donde tomará el valor de 1 si el nivel educativo del individuo es superior y 0 si cuenta con otro nivel educativo ${ }^{4}$.

X9i : secundaria, donde tomará el valor de 1 si el nivel educativo del individuo es secundaria y 0 si cuenta con otro nivel educativo.

X10i : primaria, donde tomará el valor de 1 si el nivel educativo del individuo es primaria y 0 si cuenta con otro nivel educativo.

X11i : joven, donde 1 si su edad es de 14 a 29 años y 0 si es mayor de 29 años

$\mathrm{X} 12 \mathrm{i}$ : rural, donde 1 si proviene del área rural y 0 si proviene del área urbana

$\mathrm{X} 13 \mathrm{i}$ : enfermo, donde 1 si padece de alguna enfermedad y 0 si no padece enfermedad.

En el modelo Logit se hace uso de la función de distribución acumulada logística estándar. En tal sentido, la probabilidad de que una persona obtenga un empleo informal puede ser expresada de la siguiente manera:

$$
\begin{gathered}
\operatorname{Pr}\left(y_{1}=1 \backslash X_{i}\right)=\frac{\exp \left(X_{i} \beta\right)}{1+\exp \left(X_{i} \beta\right)} \\
A\left(X_{i} \beta\right)=\frac{\exp \left(X_{t} \beta\right)}{1+\exp \left(X_{i} \beta\right)} \\
\operatorname{Pr}\left(y_{i}=1 \backslash X_{i}\right)=A\left(X_{i} \beta\right)
\end{gathered}
$$

En lo que se refiere al modelo Logit se aplicara el método de estimación de máxima verosimilitud. Se puede proceder de la siguiente manera para obtener la función de máxima verosimilitud.

Sea la probabilidad $(\zeta)$ de nuestra muestra el producto de las probabilidades de cada una de las observaciones con las que se cuenta:

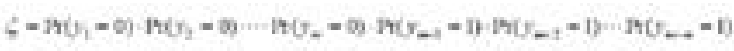

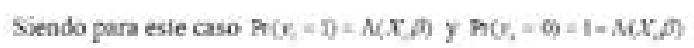

Así obtenemos la función de máxima verosimilitud para este modelo Logit el cual se representa de la siguiente manera:

$$
\begin{aligned}
& \zeta=\prod_{j=1}^{n}[1-\Lambda(X, \beta)] \cdot \prod_{i=1}^{n} \Lambda(X, \beta) \\
& \zeta=\prod_{i=1}^{n} \Lambda(X, \beta)^{N}[1-\Lambda(X, \beta)]^{-n)}
\end{aligned}
$$

Aplicamos el logaritmo natural a la función de máxima verosimilitud del modelo Logit y obtenemos la siguiente expresión:

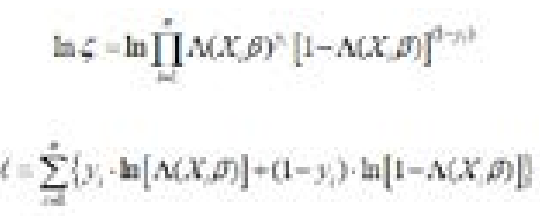

Siendo esta el logaritmo natural de la función de máxima verosimilitud, la cual se utilizará para hallar los valores de los estimadores de cada una de las variables explicativas del modelo especificado anteriormente.

\section{RESULTADOS}

En la Tabla $\mathrm{N}^{\circ} 01$ se presentan los principales indicadores laborales de la región Moquegua . Se puede notar que del total de la Población en Edad de Trabajar que se encuentra en la región Moquegua $^{5}$, aproximadamente el $74.3 \%$ está conformada por la Población Económicamente Activa, es decir, aquellas personas que se encuentran trabajando o en búsqueda de un trabajo. En lo que corresponde a la Población Económicamente Activa, el $94.9 \%$ está conformada por la población ocupada laboralmente y el restante 5.2\% lo conforma la población desocupada.

En lo que se refiere a la composición de Población Ocupada según el tipo de empleo, se puede observar que cerca del $65.6 \%$ de la población ocupada cuenta con un empleo informal y solo el $34.4 \%$ de la población ocupada cuenta con un empleo formal. Como se puede ver en la región Moquegua existe un mayor dominio de la población ocupada en el sector informal, el cual no ofrece las condiciones adecuadas para laborar. Este hecho puede tener consecuencias en el bienestar de la población ocupada debido a que un empleo informal tiene limitaciones para el trabajador.

Tabla 1: Indicadores laborales de la región Moquegua. Fuente: Elaboración propia en base a la Encuesta Nacional de Hogares 2016.

\begin{tabular}{cc}
\hline \multicolumn{2}{c}{ Población en edad de trabajar ${ }^{6}$} \\
\hline Población Económicamente Activa \\
Población Económicamente Inactiva & 74.3 \\
\hline Población económicamente activa & 25.7 \\
Ocupados & 94.9 \\
Desocupados & 5.2 \\
\hline Población ocupada & \\
Empleo Informal & 65.6 \\
Empleo Formal & 34.4 \\
\hline
\end{tabular}

\footnotetext{
${ }^{4}$ En este caso se considera a las personas que no tienen un nivel educativo dentro de la categoría "otro nivel educativo". De igual manera para la categoría "otro nivel educativo" de la variable secundaria y primaria.

${ }^{5}$ Estos indicadores fueron obtenidos del Ministerio de Trabajo y Promoción del Empleo. También se realizó el contraste mediante el cálculo de los indicadores haciendo uso de la Encuesta Nacional de Hogares modificada por la misma entidad. ${ }^{6}$ Se considera Población en edad de trabajar a la población de 14 a más años de edad.
} 
Ahora lo que se presenta a continuación es la composición del empleo informal de acuerdo a la lengua materna del trabajador, el sexo del trabajador, el sector o rama económica en la cual se desempeña el trabajador, la categoría de edad del trabajador, el área geográfica de procedencia del trabajador y la condición de salud del trabajador.

En la Tabla $\mathrm{N}^{\circ} 02$, se puede observar que el $40.3 \%$ de la población ocupada que cuenta con un empleo informal tiene como lengua materna un idioma nativo del Perú, el restante $59.7 \%$ de esta población tiene como lengua materna el castellano ó algún otro idioma extranjero.

Se puede observar que el $54.2 \%$ de la población ocupada que cuenta con un empleo de manera informal es hombre, el restante $45.8 \%$ es mujer.

El $38.6 \%$ de la población ocupada que cuenta con un empleo informal se encuentran en el sector de actividades extractivas como la agricultura, minería, ganadería y otras. E1 $30.5 \%$ de la población con empleo informal se encuentra en el sector de servicios. En tercer lugar, el sector comercio posee el $16.1 \%$ de la población ocupada con empleo informal.

El $73.3 \%$ de la población que cuenta con un empleo informal es adulto. El restante $26.7 \%$ de la población con empleo informal es joven.

Podemos ver que, de acuerdo al área geográfica, el 69.2\% de la población que cuenta con un empleo informal proviene del sector urbano y el restante $30.8 \%$ corresponde al área rural.

En la misma tabla se puede observar que de la población ocupada que cuenta con un empleo informal, $54.4 \%$ cuenta con una enfermedad y el restante $45.6 \%$ no cuenta con enfermedad.

Tabla 2: Descripción de variables. Fuente: Elaboración propia en base a la Encuesta Nacional de Hogares 2016.

\begin{tabular}{|c|c|c|c|}
\hline Nativo $==1$ & Empleo Informal & Empleo formal & Total \\
\hline Nativo & 40.3 & 21.9 & 34.0 \\
\hline Otros & 59.7 & 78.1 & 66.0 \\
\hline Mujer $==1$ & Empleo informal & Empleo formal & Total \\
\hline Hombre & 54.2 & 71.5 & 60.2 \\
\hline Mujer & 45.8 & 28.6 & 39.8 \\
\hline Rama de actividad económica & Empleo informal & Empleo formal & Total \\
\hline Extractivas & 38.6 & 7.5 & 27.9 \\
\hline Industria & 5.7 & 6.6 & 6.0 \\
\hline Construcción & 7.8 & 10.6 & 8.8 \\
\hline Comercio & 16.1 & 10.4 & 14.1 \\
\hline Servicios & 30.5 & 64.5 & 42.2 \\
\hline Hogares & 1.3 & 0.3 & 1.0 \\
\hline Nivel educativo culminado & Empleo informal & Empleo formal & Total \\
\hline Sin nivel educativo & 15.9 & 3.0 & 11.5 \\
\hline Primaria & 27.8 & 10.7 & 21.9 \\
\hline Secundaria & 40.8 & 34.3 & 38.5 \\
\hline Superior & 15.5 & 24.7 & 15.0 \\
\hline Joven $==1$ & Empleo informal & Empleo formal & Total \\
\hline Joven & 26.7 & 16.4 & 23.1 \\
\hline Adulto & 73.3 & 83.7 & 76.9 \\
\hline Rural $==1$ & Empleo informal & Empleo formal & Total \\
\hline Urbano & 69.2 & 90.3 & 76.5 \\
\hline Rural & 30.8 & 9.7 & 23.5 \\
\hline Enfermedad & Empleo informal & Empleo formal & Total \\
\hline No Enfermedad & 45.6 & 42.8 & 44.6 \\
\hline Enfermedad & 54.4 & 57.2 & 55.4 \\
\hline
\end{tabular}

En Tabla $N^{\circ} 03$ se muestran los resultados del modelo de regresión estimado para la muestra seleccionada en la región Moquegua. En la primera columna se observan las variables del modelo, en la segunda columna tenemos los valores hallados de los coeficientes de las variables, en la tercera columna se presentan los errores estándar robustos, en la cuarta columna se muestra el p-value y en la quinta y sexta columna tenemos el intervalo de confianza inferior y superior respectivamente.

Observando el p-value de los coeficientes del modelo que prueba la hipótesis nula de que dicho coeficiente no influye significativamente en la variable dependiente, tenemos que los coeficientes de la variable nativo, hogar, primaria y enfermo son no significativos, pues al nivel de significancia del 5\% dichas hipótesis no son rechazadas.

Cabe señalar los coeficientes del modelo de regresión aplicado expresan el cambio en el logaritmo de las probabilidades cuando la variable explicativa cambia en una unidad (Gujarati, 2010). Por tal motivo antes de realizar cualquier interpretación sobre dichos coeficientes es recomendable aplicar una transformación. 
Estos resultados muestran que el valor del coeficiente de .9562788 significa que una mujer incrementa su probabilidad de conseguir un empleo informal en 0.95 veces con respecto a un varón, manteniendo las demás variables constantes. En el caso del coeficiente de -1.895696 significa que una persona que trabaja en el sector servicios disminuye su probabilidad de obtener un empleo informal en -1.89 veces, con respecto a las personas que trabajan en otros sectores, manteniendo todas las demás variables constantes.

Con respecto al sector o rama de actividad laboral. El coeficiente de -1.437209 significa que una persona que labora en el sector comercio disminuye su probabilidad de obtener un empleo informal en -1.43 veces, con respecto a las personas que trabajan en otros sectores. El coeficiente de -1.296937 significa que una persona que labora en el sector construcción disminuye la probabilidad de obtener un empleo informal en -1.29 veces, con respecto a las personas que laboran en otros sectores de la actividad económica. El coeficiente de -1.458056 indica que una persona que labora en el sector industria disminuye la probabilidad de obtener un empleo informal en -1.45 veces, con respecto a las personas que laboran en otras ramas de actividad.

Por el lado de la educación se tiene el coeficiente -2.439419 que indica que las personas con nivel educativo superior disminuyen su probabilidad de hallar un empleo informal en -2.43 veces con respecto a personas con otro nivel educativo. En el caso del coeficiente -1.248683 , este indica que personas con nivel educativo secundario disminuyen su probabilidad de encontrar un empleo informal en -1.24 veces con respecto a personas que cuentan con otro nivel educativo.

Considerando la edad, el coeficiente de 1.246111 significa que el hecho de ser joven, incrementa la probabilidad de encontrar un empleo informal en 1.24 veces con respecto a los adultos.

Si también consideramos elárea geográfica de procedencia de las personas, el coeficiente de 1.079233 significa que personas que provienen del área rural incrementan tu probabilidad de encontrar un empleo informal en 1.07 veces con respecto a las personas que provienen del área urbana.

Tabla 3: Resultados del modelo de regresión

\begin{tabular}{|c|c|c|c|c|c|c|}
\hline Informal & Coef. & Robust Std. Err. & $\mathrm{z}$ & $\mathrm{P}>\mathrm{Z}$ & {$[95 \%$ Conf. } & Interval] \\
\hline Nativo & 0.2531157 & 0.1530604 & 1.65 & 0.098 & -0.0468772 & 0.5531085 \\
\hline Mujer & 0.9562788 & 0.131412 & 7.28 & 0 & 0.698716 & 1.213842 \\
\hline Hogar & -0.371523 & 0.8915038 & -0.42 & 0.677 & -2.118838 & 1.375792 \\
\hline Servicio & -1.895696 & 0.1920724 & -9.87 & 0 & -2.272151 & -1.519241 \\
\hline Comercio & -1.437209 & 0.2368547 & -6.07 & 0 & -1.901436 & -0.9729828 \\
\hline Construccion & -1.296937 & 0.2610024 & -4.97 & 0 & -1.808492 & -0.7853813 \\
\hline Industria & -1.458056 & 0.2649971 & -5.5 & 0 & -1.97744 & -0.9386709 \\
\hline Superior & -2.439419 & 0.2820857 & -8.65 & 0 & -2.992296 & -1.886541 \\
\hline Secundaria & -1.248683 & 0.2746014 & -4.55 & 0 & -1.786892 & -0.7104744 \\
\hline Primaria & -0.5061503 & 0.2900535 & -1.75 & 0.081 & -1.074645 & 0.0623441 \\
\hline Joven & 1.246111 & 0.1550692 & 8.04 & 0 & 0.9421808 & 1.550041 \\
\hline Rural & 1.079233 & 0.2294227 & 4.7 & 0 & 0.6295731 & 1.528893 \\
\hline Enfermo & -0.184077 & 0.127181 & -1.45 & 0.148 & -0.4333471 & 0.0651931 \\
\hline Constante & 2.553967 & 0.3068149 & 8.32 & 0 & 1.952621 & 3.155313 \\
\hline
\end{tabular}

Como se ha mencionado anteriormente, el modelo logit se utiliza para modelar la probabilidad de que $\mathrm{y}_{-} \mathrm{i}=1$ dado ciertos valores de $\mathrm{X}$, que representa la función de distribución acumulada para una función de distribución logística. Dicha función es evaluada en $z=X \_i \beta$, de la siguiente manera:

$$
\begin{gathered}
\operatorname{Pr}\left(y_{i}=1 \backslash X_{i}\right)=F\left(X_{i} \beta\right) \\
F\left(X_{i} \beta\right)=\frac{1}{1+e^{-\left(X_{1} \beta\right)}} \\
F\left(X_{i} \beta\right)=\frac{1}{1+e^{-\left(\hat{\beta}_{0}+\beta_{1} X_{1}+\cdots \beta_{R} X_{k}\right)}}
\end{gathered}
$$

Para nuestro caso solo consideraremos las variables que resultaron significativas en nuestro modelo planteado. Así mismo nos referiremos a las características más sobresalientes de la población que se encuentra laborando. Consideraremos a un individuo hombre, que se encuentra laborando en el sector servicios, que cuenta con estudios secundarios, que es adulto y procede del área urbana.

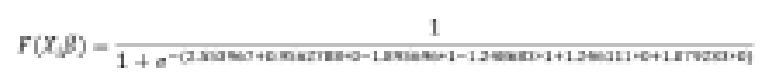

Para dicho individuo se encuentra que la probabilidad obtener un empleo informal es de 0.3565403 . Por el contrario si consideramos a un individuo que sea mujer, labore en el sector servicios, con estudios secundarios, joven y que procede del área rural podremos hallar una probabilidad de 0.93650601 de obtener un empleo informal. 


\section{CONCLUSIONES}

Se puede concluir que los factores socioeconómicos que determinan el empleo informal en la región Moquegua son la condición de mujer del individuo, laborar en el sector servicios, comercio, construcción e industria, contar con estudios superiores y secundarios, ser joven y proceder del área rural.

En el caso de la variable que caracteriza la condición de mujer del individuo, los resultados indican que las mujeres tienen una mayor probabilidad de caer en el empleo informal en comparación con los hombres. El sector o rama de actividad económica donde labora un individuo como son el sector servicios, comercio, construcción e industria, también resultan ser determinantes en el empleo informal. En este caso todos estos sectores disminuyen la probabilidad de formar parte del empleo informal. El nivel educativo alcanzado como el superior y el secundario también resultan ser determinantes en el empleo informal en la región Moquegua. Ambos niveles educativos disminuyen la probabilidad del encontrar un empleo informal. La edad es considerada otro factor determinante del empleo informal ya que los jóvenes aumentan su probabilidad de formar parte del empleo informal con respecto a los adultos. Así mismo también se observa que el área geográfica es otro factor determinante del empleo informal. En este caso proceder del área rural aumenta la probabilidad de formar parte del empleo informal en comparación con alguien que procede del área urbana.

Como se ha podido observar en el ejemplo de muestra, ser mujer, ser del área rural y joven representa un incremento sustancial en la probabilidad de obtener un empleo informal en la región Moquegua.

\section{REFERENCIAS BIBLIOGRÁFICAS}

1. Ayala, A. (2014). Indicadores sociales de desigualdad en base a la ENAHO con Stata. Universidad Nacional mayor de San Marcos.

2. Banco Mundial. (2007). Informalidad: escape y exclusión. Banco Mundial.

3. Cameron, C., \& Trivedi, P. (s.f.). Microefconometrics Methods and applications. Cambridge University Press.

4. CEPLAN. (2016). Economia informal en el Perú: Situación actual y perspectivas.

5. Gujarati, D. N. (2010). Econometria.

6. Hosmer, D., \& Lemeshow, S. (s.f.). Applied logistic regression. Library of congress cataloging in publication data.

7. INEI. (2014). Producción y empleo informal en el Perú, cuenta satelite del empleo informal 2007 - 2012.

8. Jhonton, J., \& Dinardo, J. (s.f.). Econometrics Methods. Mc Grau-Hill.

9. Larrañaga, P., Inza, I., \& Moujahid, A. (s.f.). Regresion Logistica. Universidad del País Vasco.

10. Leasaski, D. A., \& Crispín, Á. R. (2010). La oferta laboral juvenil de la region moquegua. Ministerio de trabajao.

11. Millones, E. A. (2003). Modelos Probit y Tobit aplicados al estudio de la oferta. Universidad Nacional de San Marcos.

12. Millones, E. A. (2003). Modelos Probit y Tobit aplicados al estudio de la oferta.

13. Mora, J. J., \& Ulloa, M. P. (s.f.). El efecto de la educación sobre la calidad del empleo en Colombia. Universidad Icesi.

14. MTPE. (2016). Informe anual del empleo en el Perú 2016.

15. O'Halloran, S. (s.f.). Sustainable Development U9611 Econometrics II.

16. Orlando moscote flórez, W. A. (2012). Modelos Logit y Probit: Un caso de aplicación. Universidad de santo Tomas.

17. Stock, J. H., \& Watson, M. M. (2012). Introducción a la Econometria.

18. Vera, N. P., Gil, J. F., Chavez, I. E., \& Manayay, D. T. (2015). Informe Anual del Empleo en el Perú. 